\title{
XIX Congrès de la Société d'Andrologie de Langue Française
}

\section{Centre International des Conférences de GENEVE (CICG) 12-13-14 Décembre 2002}

Comité d'Organisation: Hervé LUCAS

Aldo CAMPANA

Georges Antoine de BOCCARD

Gabriel de CANDOLLE

Francesco BIANCHI DEMICHELI

Blaise BOURRIT

Comité Scientifique : $\quad$ Stylianos ANTONARAKIS

Serge CARREAU

Didier CHARDONNENS

Christophe ISELIN

Christophe A. MEIER

Willy PASINI

Jacques PHILIPPE

François PRALONG

Charles SULTAN

Preprogrammme

Jeudi 12 Décembre 2002

\section{LES TRAITEMENTS DU CANCER PROSTATIQUE : DIFFERENTES TECHNIQUES ET PRONOSTIC} SEXUEL.

- Le traitement chirurgical " classique " : technique et pronostic sexuel.

- Le traitement chirurgical par laparoscopie : technique et pronostic sexuel.

- Le traitement par radiothérapie interstitielle (Brachythérapie) : technique et pronostic sexuel. 
- Le traitement par radiothérapie externe : technique et pronostic sexuel.

- Le traitement par ultrasons (Ablatherm) : technique et pronostic sexuel.

- Evaluation sexologique : Analyse des séries internationales, synthèse et discussion.

\section{GENETIQUE DE L'INFERTILITE MASCULINE.}

- Facteurs impliqués dans le remodelage de la chromatine au cours de la spermiogenèse.

- Anomalies chromosomiques et infertilité masculine.

- Anomalies géniques et infertilité masculine.

- La méïose : Modèles d'étude et nouveaux acquis.

\section{LE DIAGNOSTIC PRE-IMPLANTATOIRE (DPI) D'INDICATION MASCULINE}

- Qu'est ce que le DPI.

- Tour d'europe du DPI

- Indications andrologiques : quelle démarche pour en arriver au DPI

- La place du conseil génétique

\section{APOPTOSE, SPERMATOGENESE ET SPERMATOZOIDES}

- Mécanismes moléculaires intervenant dans l'apoptose

- BARD-1, spermatogénèse et apoptose

- Apoptose et spermatozoïdes

- Apoptose : implication dans les troubles de la spermatogenèse

\section{DIAGNOSTICS EN ANDROLOGIE}

Signification et traitement des anticorps anti-spermatozoides dans la glaire, le sang, et le plasma séminal.

- Indications pour la recherche d' anticorps anti-spermatozoïdes.

- Techniques et interprétation des résultats.

- Perspectives diagnostiques et thérapeutiques.

Le point sur les marqueurs de la spermatogenèse.

- L'Hormone AntiMullérienne (AMH), marqueur séminal de la spermatogenèse ?

- Le dosage de l'inhibine B et de la FSH dans les troubles de la spermatogenèse.

\section{LES ENFANTS DE L'ICSI.}

- Analyse épidémiologique et critique de la littérature.

- L'avis du pédiatre

- Que doit dire le conseil génétique avant ICSI 


\section{Vendredi 13 Décembre 2002}

INTERNATIONAL SYMPOSIUM : ESTROGENES ET FONCTION DE REPRODUCTION CHEZ LE MALE

- Les sources d'estrogène.

- Estrogènes et contrôle de la prolifération germinale.

- Discussion avec les orateurs.
- Testostérone et os.

- Androgen deficiency in HIV and other wasting conditions (TS).
- Receptors in primates and human (TS).

- The lack of estrogens in men : clinical aspects.

- La DHEA chez l'homme.

\section{Samedi 14 Décembre 2002}

\section{DYSFONCTIONS SEXUELLES MASCULINES : UNE APPROCHE MULTIDISCIPLINAIRE}

- Actualité sur les nouvelles molécules dans le traitement des dysfonctions érectiles.

- Est-ce que traiter l'érection signifie guérir l'impuissance.

- Rêves, fantasmes érotiques et androgènes.

- Dysfonctions sexuelles masculines et trouble de la personnalité

- Ejaculation précoce, érection instable, trouble du désir, symptome ou crise de la masculinité.

\section{SYMPOSIUM Satellite}

- La partenaire des hommes souffrant d'un dysfonctionnement érectile : à propos d'une recherche

- Présentation de l'étude internationale PFIZER sur la qualité de vie des 40-80 ans

\section{SYMPOSIUM Satellite}

- Les déterminants androgéniques de la sexualité :

- La sphère urogénitale

- Le cerveau

- Synthèse Clinique

LA S.A.L.F. soutient la recherche en ANDROLOGIE :

Presentations des laureats des bourses de recherche de la S.A.L.F.

Presentation orale des memoires des prix de DEA, de DESS de biologie de la reproduction et de DESC d'andrologie.

Communications orales selectionnées primées

Posters selectionnés primés

\section{Renseignements :}

Hervé LUCAS, Maternité de I'Hôpital Cantonal Universitaire de Genève, 30 Boulevard de la Cluse, 1211 GENEVE 14

Tél: 0041223824332 - Fax: 0041223824385 Mail: herve.lucas@hcuge.ch 